\title{
Impact of Clinical Specialty Setting and Geographic Regions on Disease Management in Patients with Psoriatic Arthritis in the United States: A Multicenter Observational Study
}

\author{
Phillip J. Mease ${ }^{1}$ Clive Liu ${ }^{2} \cdot$ Evan Siegel $^{3} \cdot$ Heather Richmond ${ }^{4} \cdot$ Meijing Wu$^{5} \cdot$ Liang Chen $^{5} \cdot$ Kevin Douglas $^{5}$. \\ Benjamin Lockshin ${ }^{6}$
}

Published online: 14 October 2019

(C) The Author(s) 2019

\begin{abstract}
Background Information on the factors that influence treatment management decisions for psoriatic arthritis (PsA) is limited. Objective Our objective was to evaluate the impact of clinical specialty setting and geographic region on the management of patients with PsA in the USA.

Methods LOOP was a multicenter, cross-sectional, observational study conducted across 44 sites in the USA. Patients were aged $\geq 18$ years with a suspected or established diagnosis of PsA and were routinely visiting a rheumatologist or dermatologist. All patients enrolled in the study were assessed by both a rheumatologist and a dermatologist. Primary outcomes were the times from symptom onset to PsA diagnosis; PsA diagnosis to first conventional synthetic disease-modifying antirheumatic drug (csDMARD); PsA diagnosis to first biologic DMARD (bDMARD); and first csDMARD to first bDMARD.

Results Of 681 patients enrolled in the study, 513 had a confirmed diagnosis of PsA and were included in this analysis. More patients were recruited by rheumatologists $(71.3 \%)$ than by dermatologists $(28.7 \%)$. The median time from symptom onset to diagnosis of PsA was significantly shorter for patients enrolled by rheumatologists than for those enrolled by dermatologists ( 1.0 vs. 2.6 years; $p<0.001)$. Disease activity and burden were generally similar across enrolling specialties. However, patients in western areas of the USA had less severe disease than those in central or eastern areas, including measures of joint involvement, enthesitis, and dactylitis.

Conclusions There was a substantial delay in the time from symptom onset to diagnosis in this study population, and this was significantly longer for patients enrolled in the dermatology versus the rheumatology setting. This supports the need for collaboration across specialties to ensure faster recognition and treatment of PsA.
\end{abstract}

Electronic supplementary material The online version of this article (https://doi.org/10.1007/s40257-019-00470-6) contains supplementary material, which is available to authorized users.

\section{Phillip J. Mease}

pmease@philipmease.com

1 Swedish Medical Center, Providence St. Joseph Health, and University of Washington, Seattle, WA, USA

2 Bellevue Dermatology Clinic, Bellevue, WA, USA

3 Arthritis and Rheumatism Associates and Georgetown University, Rockville, MD, USA

4 Clear Dermatology, Houston, TX, USA

5 AbbVie Inc., North Chicago, IL, USA

6 US Dermatology Partners, Rockville, MD, USA

\section{Key Points}

LOOP is the first study to assess the impact of clinical specialty setting and geographic region of the USA on the diagnosis and management of patients with psoriatic arthritis (PsA).

The median time from symptom onset to diagnosis of PsA was significantly shorter for patients enrolled by rheumatologists than for those enrolled by dermatologists.

Patients enrolled from western regions of the USA tended to have less severe disease than those from eastern and central regions. 


\section{Introduction}

Psoriatic arthritis (PsA) is a primarily peripheral inflammatory joint disease that occurs in up to $30 \%$ of patients with psoriasis and can result in functional disability and reduced quality of life [1-3]. The historical Moll and Wright classification criteria identified five clinical subtypes of joint involvement in PsA: oligoarticular, polyarticular, distal, arthritis mutilans, and axial [2, 4, 5]. Patients can also present with a variety of other clinical manifestations, including enthesitis, dactylitis, and nail dystrophy as well as comorbidities such as uveitis, inflammatory bowel disease, metabolic syndrome (obesity, hypertension, and hyperlipidemia), diabetes mellitus, and osteoporosis $[4,6]$. The type and pattern of musculoskeletal involvement, clinical characteristics, and comorbidities can vary widely between patients or within the same patient over time. The heterogeneity of PsA therefore presents a diagnostic challenge, and patients may present to and be primarily managed by different clinical specialties, including rheumatology and dermatology [2].

Early diagnosis and treatment of PsA is essential to prevent long-term structural joint damage and functional disability [7-9]. The American College of Rheumatology/ National Psoriasis Foundation, the European League Against Rheumatism (EULAR), and the Group for Research and Assessment of Psoriasis and Psoriatic Arthritis (GRAPPA) recommend treatment with conventional synthetic disease-modifying antirheumatic drugs (csDMARDs), such as methotrexate or sulfasalazine, and/or biologic agents (bDMARDs) such as tumor necrosis factor inhibitors or interleukin-12/23 inhibitors [10-12]. Although evidence is lacking for the ability of csDMARDs to inhibit structural damage in PsA [11, 13-17], effective inhibition has been demonstrated by bDMARDs [11, 14, 18, 19]. EULAR and GRAPPA also recommend a multidisciplinary approach to the treatment of PsA, with multispecialty assessment and management $[10,11]$.

Information on the factors that influence treatment management decisions for PsA is limited. The objective of this analysis was to investigate the impact of disease characteristics, clinical specialty setting, and geographic region on the diagnosis and management of patients with PsA. Disease activity and disease burden were also investigated across clinical specialty setting and geographic region.

\section{Materials and Methods}

\subsection{Study Design and Patients}

LOOP was a multicenter, cross-sectional, observational study conducted across 44 sites in the USA. Patients were aged $\geq 18$ years with a suspected or established diagnosis of PsA and were routinely visiting a rheumatologist or a dermatologist (Fig. 1 in the Electronic Supplementary Material [ESM]). All patients enrolled in the study were assessed by both a rheumatologist and a dermatologist, and patients with a diagnosis of PsA confirmed by the rheumatologist were included in the present analysis.

\subsection{Assessments}

The primary outcomes were the time from inflammatory musculoskeletal symptom onset to PsA diagnosis; time from PsA diagnosis to first csDMARD; time from PsA diagnosis to first bDMARD; and time from first csDMARD to first bDMARD. Secondary outcomes included the current disease activity and disease burden as measured by clinical assessments (tender joint count in 68 joints, swollen joint count in 66 joints, Leeds Enthesitis Index [LEI], dactylitis count, Bath Ankylosing Spondylitis Disease Activity Index [BASDAI], psoriasis body surface area [BSA], psoriatic nail count, and Psoriasis Area and Severity Index [PASI]) and patient-reported outcomes (Health Assessment Questionnaire-Disability Index [HAQ-DI], Short Form 12-item health survey version 2.0 [SF12v2], Dermatology Life Quality Index [DLQI], and Patient Global Assessment of disease [PtGA]).

\subsection{Statistical Analysis}

Demographics of the study population are presented by enrolling specialty and geographic region (eastern, western, or central USA; see the ESM for further details) using descriptive statistics. The impact of clinical specialty setting and geographic region on time from symptom onset to PsA diagnosis was assessed using Kaplan-Meier analysis and a Cox proportional hazards model, with time as the response variable and clinical specialty or geographic region as the independent variable. Disease activity and disease burden were compared between clinical specialty using a twosample $t$ test and across geographic region using one-way analysis of variance.

\section{Results}

\subsection{Patients and Demographics}

A total of 681 patients were enrolled in the LOOP study, 513 of whom had a diagnosis of PsA confirmed by a rheumatologist and were included in this analysis. Of these, 404 patients had their PsA diagnosis established prior to study entry (established PsA), whereas 109 patients had their PsA diagnosis confirmed during the study (suspected PsA). The 
Fig. 1 Distribution of time from symptom onset to psoriatic arthritis diagnosis. Percentages are calculated on non-missing values. Full analysis set

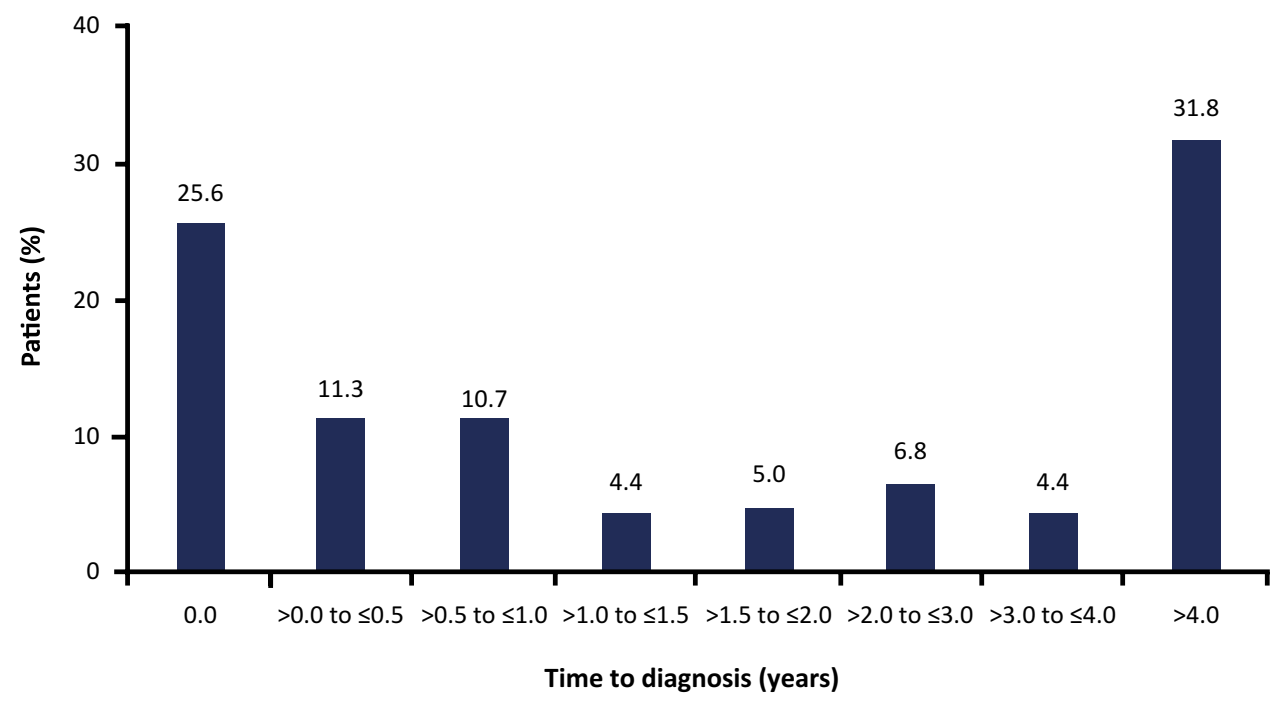

Table 1 Demographics and disease characteristics of patients categorized by the specialty of the enrolling physician and geographic region of the USA

\begin{tabular}{|c|c|c|c|c|c|c|}
\hline \multirow[t]{2}{*}{ Characteristic } & \multicolumn{2}{|c|}{ Specialty setting } & \multicolumn{3}{|l|}{ Geographic region } & \multirow[t]{2}{*}{ Overall $(n=513)$} \\
\hline & $\begin{array}{l}\text { Rheumatology } \\
(n=366)\end{array}$ & Dermatology $(n=147)$ & Eastern $(n=348)$ & Central $(n=94)$ & Western $(n=71)$ & \\
\hline Age, years & $55.9 \pm 13.5$ & $53.1 \pm 11.8$ & $56.3 \pm 12.7$ & $53.7 \pm 12.6$ & $51.0 \pm 14.6$ & $55.1 \pm 13.1$ \\
\hline Sex, male ${ }^{a}$ & $151(41.3)$ & $68(46.3)$ & $134(38.5)$ & $44(46.8)$ & $41(57.7)$ & $219(42.7)$ \\
\hline \multicolumn{7}{|l|}{ Race $^{\mathrm{b}}$} \\
\hline White & 339 (92.6) & $128(87.1)$ & $324(93.1)$ & 85 (90.4) & $58(81.7)$ & $467(91.0)$ \\
\hline Black & $14(3.8)$ & $11(7.5)$ & 17 (4.9) & $7(7.4)$ & $1(1.4)$ & $25(4.9)$ \\
\hline Asian & $6(1.6)$ & $7(4.8)$ & $3(0.9)$ & $2(2.1)$ & $8(11.3)$ & $13(2.5)$ \\
\hline Other & $3(0.8)$ & $1(0.7)$ & $4(1.1)$ & 0 & 0 & $4(0.8)$ \\
\hline Multiple & $4(1.1)$ & 0 & 0 & 0 & $4(5.6)$ & $4(0.8)$ \\
\hline Weight, kg & $89.2 \pm 21.5$ & $90.0 \pm 20.1^{\mathrm{b}}$ & $89.1 \pm 20.6$ & $93.4 \pm 20.9$ & $85.4 \pm 23.5^{\mathrm{b}}$ & $89.4 \pm 21.1^{\mathrm{b}}$ \\
\hline BMI & $31.4 \pm 7.3$ & $31.8 \pm 6.7^{\mathrm{b}}$ & $31.9 \pm 7.0$ & $32.2 \pm 7.3$ & $28.8 \pm 7.3^{\mathrm{b}}$ & $31.5 \pm 7.2^{\mathrm{b}}$ \\
\hline
\end{tabular}

Data are presented as mean \pm standard deviation or $N(\%)$ unless otherwise indicated

$B M I$ body mass index

${ }^{\text {a}}$ Percentages calculated on non-missing values

${ }^{b}$ Data missing for one patient

majority of patients (57.3\%) were female, with a mean age of 55.1 years and a mean body mass index of 31.5 (Table 1). Over twice as many patients were recruited by rheumatologists $(71.3 \%)$ than by dermatologists $(28.7 \%)$. More patients were recruited from the eastern regions of the USA $(67.8 \%)$ than central (18.3\%) and western $(13.8 \%)$ regions, which was proportional to the number of study sites in each region (see the ESM). Demographics were similar across clinical specialty setting and geographic region (Table 1).

\subsection{Treatment Patterns in the Overall Population}

In the overall population, the median time from symptom onset to diagnosis of PsA was 1.2 years, with almost onethird of patients $(31.8 \%)$ waiting $>4$ years for a diagnosis (Fig. 1). The median time from PsA diagnosis to first csDMARD and bDMARD was 1.0 and 2.4 years, respectively. In total, $282(55.0 \%)$ and $354(69.0 \%)$ patients received csDMARDs and bDMARDs, respectively. Prior to receiving a diagnosis of PsA, similar proportions of patients received therapy with csDMARDs (20.7\%) and bDMARDs (23.6\%). After diagnosis, the number of patients receiving these treatments increased, and a higher proportion of 
Dermatologist Rheumatologist

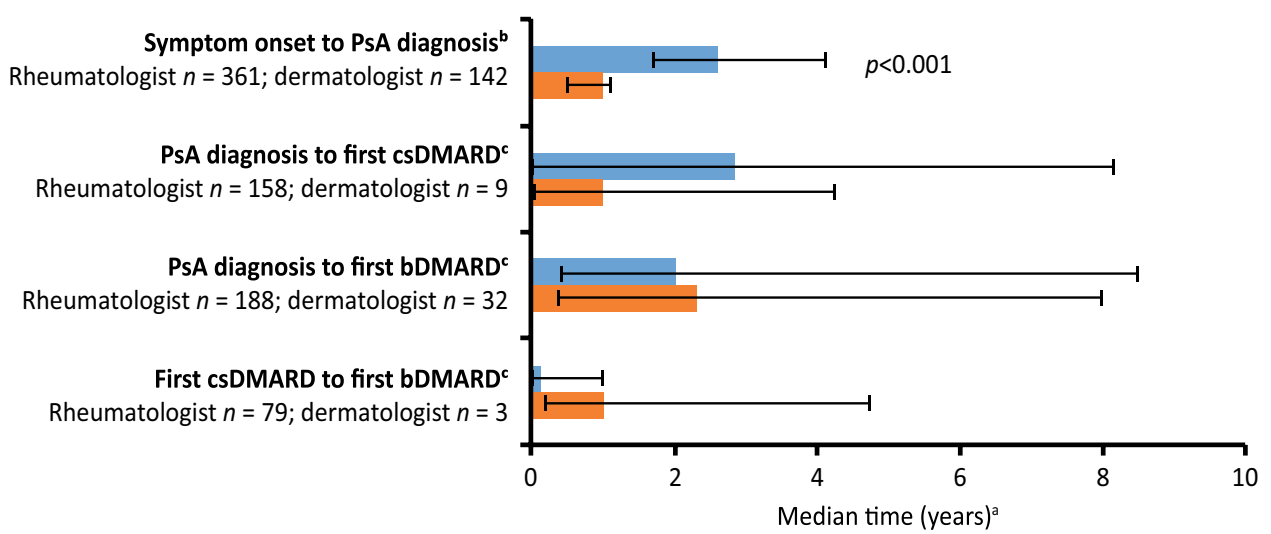

Fig. 2 Timing of disease management steps by clinical specialty setting. $* p$ value calculated using a Cox proportional hazards model, with time as a response variable and specialty as an independent variable. $p$ values are not shown for the other three parameters because some data were missing. Error bars are $95 \%$ confidence intervals for symptom onset to psoriatic arthritis diagnosis and interquartile range for the remaining parameters. ${ }^{a}$ Estimated from Kaplan-Meier analyses. ${ }^{\mathrm{b}}$ Full analysis set. ${ }^{\mathrm{c} E s t a b l i s h e d ~ p a t i e n t s ~ o n l y . ~}$ DDMARD biologic disease-modifying antirheumatic drug, $\operatorname{cs} D M A R D$ conventional synthetic disease-modifying antirheumatic drug, PSA psoriatic arthritis patients received therapy with bDMARDs $(45.4 \%)$ versus csDMARDs (34.3\%). A total of $73(14.2 \%)$ patients did not receive treatment with csDMARDs or bDMARDs (although this included newly diagnosed patients who may not have started treatment, and in some cases these data may have been missing from patient records).

The majority of patients with a confirmed diagnosis of PsA presented with skin symptoms first (63.0\%), with smaller proportions presenting with simultaneous skin and joint symptoms $(21.1 \%)$ or joint symptoms first (14.2\%). Patients who presented with joint symptoms first experienced a longer median time to PsA diagnosis (3.32 years) than those presenting with skin symptoms first ( 0.76 years) or simultaneous joint and skin symptoms ( 0.38 years).

\subsection{Effect of Clinical Specialty Setting on the Management of PsA}

Patients enrolled by rheumatologists had a significantly shorter median time from symptom onset to diagnosis of PsA than those enrolled by dermatologists ( 1.0 vs. 2.6 years; hazard ratio 1.47 ; $95 \%$ confidence interval 1.20-1.78; $p<0.001$; Fig. 2). Numerical differences were seen between enrolling specialties in time to first csDMARD, time to first bDMARD, and time from csDMARD to bDMARD. However, these results should be interpreted with caution as data were not available for all patients.

Disease activity and disease burden were generally similar for patients enrolled by rheumatologists and dermatologists (Table 2). However, patients enrolled by dermatologists had significantly higher skin scores (as assessed by BSA and PASI), significantly higher enthesitis scores (as measured by
LEI), and significantly worse quality of life (as measured by DLQI) than those enrolled by rheumatologists. In addition, patients enrolled by rheumatologists presented with a more severe average PtGA score at the time of diagnosis than those enrolled by dermatologists (Table 2).

\subsection{Effect of Geographic Region on the Management of PsA}

Numerical differences were observed in the time from symptom onset to PsA diagnosis and time from PsA diagnosis to first csDMARD and bDMARD between geographic regions, but these were not significant. Patients enrolled in eastern and western regions of the USA experienced similar median times from symptom onset to PsA diagnosis (1.0 and 1.1 years, respectively) and from PsA diagnosis to first csDMARD (2.8 and 3.3 years, respectively). In comparison, patients enrolled in central regions had a numerically longer median time from symptom onset to PsA diagnosis (1.8 years) and a shorter time from PsA diagnosis to first csDMARD and bDMARD (1.6 and 1.9 years, respectively). The time from PsA diagnosis to first bDMARD was slightly longer for patients enrolled from eastern regions (3.1 years) than for those from western and central regions (2.1 and 1.9 years, respectively).

Patients enrolled from western regions tended to have less severe disease than those from eastern and central regions (Table 3). This was observed in measures of joint involvement such as tender joint count and swollen joint count, as well as enthesitis score (LEI), dactylitis count, and BASDAI. This translated to improved quality-of-life scores in western patients as assessed by PtGA (at both time of diagnosis and 
Table 2 Current disease activity and disease burden by clinical specialty setting

\begin{tabular}{lccr}
\hline Measure & $\begin{array}{l}\text { Rheuma- } \\
\text { tologist } \\
(n=366)\end{array}$ & $\begin{array}{l}\text { Derma- } \\
\text { tologist } \\
(n=147)\end{array}$ & $p$ value $^{\mathrm{a}}$ \\
\hline TJC68 & $8.2 \pm 10.8$ & $9.9 \pm 10.3$ & 0.095 \\
SJC66 & $3.4 \pm 6.4$ & $4.3 \pm 6.0$ & 0.154 \\
Enthesitis based on LEI & $1.0 \pm 1.6$ & $1.5 \pm 1.8$ & $\mathbf{0 . 0 0 5}$ \\
Dactylitis count & $0.6 \pm 1.5$ & $0.8 \pm 1.7$ & 0.241 \\
BASDAI & $4.6 \pm 3.2^{\mathrm{b}}$ & $4.3 \pm 3.3^{\mathrm{c}}$ & 0.248 \\
BSA (\%) & $5.0 \pm 10.9$ & $8.1 \pm 14.5$ & $\mathbf{0 . 0 2 4}$ \\
Psoriatic nail count & $1.9 \pm 3.1^{\mathrm{d}}$ & $2.0 \pm 3.1$ & 0.734 \\
PASI & $3.0 \pm 5.1^{\mathrm{e}}$ & $5.5 \pm 7.9^{\mathrm{f}}$ & $<\mathbf{0 . 0 0 1}$ \\
PtGA at the time of diagnosis & $6.7 \pm 2.7^{\mathrm{g}}$ & $5.9 \pm 3.1^{\mathrm{h}}$ & $\mathbf{0 . 0 0 3}$ \\
PtGA during last visit & $4.7 \pm 2.9^{\mathrm{i}}$ & $4.7 \pm 3.0^{\mathrm{j}}$ & 0.976 \\
HAQ-DI & $0.9 \pm 0.7$ & $0.7 \pm 0.7$ & 0.057 \\
SF12v2 PCS & $40.6 \pm 10.5^{\mathrm{g}}$ & $41.8 \pm 10.3$ & 0.244 \\
SF12v2 MCS & $46.9 \pm 11.1^{\mathrm{g}}$ & $47.9 \pm 11.9$ & 0.361 \\
DLQI & $5.4 \pm 5.9^{\mathrm{b}}$ & $7.8 \pm 7.4$ & $<\mathbf{0 . 0 0 1}$ \\
\hline
\end{tabular}

Bold text indicates significant differences

Data are presented as mean \pm standard deviation unless otherwise indicated

BASDAI Bath Ankylosing Spondylitis Disease Activity Index, BSA body surface area with psoriasis, DLQI Dermatology Life Quality Index, $H A Q-D I$ Health Assessment Questionnaire-Disability Index, LEI Leeds Enthesitis Index, MCS Mental Component Score, PASI Psoriasis Area and Severity Index, PCS Physical Component Score, PtGA Patient Global Assessment of disease, SF12v2 Short Form 12-item health survey version 2.0, SJC66 swollen joint count in 66 joints, TJC68 tender joint count in 68 joints

${ }^{a} p$ value from two-sample $t$ test: rheumatologist vs. dermatologist

${ }^{\mathrm{b}} n=361$

${ }^{c} n=144$

$\mathrm{d}_{n=321}$

${ }^{\mathrm{e}} n=314$

${ }^{\mathrm{f}} n=145$

${ }^{\mathrm{g}} n=365$

$\mathrm{h}_{n}=138$

${ }^{\mathrm{i}} n=341$

${ }^{\mathrm{j}} n=65$

last visit) as well as physical function measured by HAQ-DI and the SF12v2 Physical Component Score. However, measures of skin involvement were similar between the regional groups, as was DLQI.

\section{Discussion}

LOOP is the first study to assess the impact of clinical specialty setting and geographic region of the USA on the diagnosis and management of patients with PsA. Our results showed that specialty setting and geographic region were both associated with differences in the timing and choice of disease management steps, as well as disease activity and burden, in patients with PsA.

A key finding of this study was that, irrespective of clinical setting, there was a delay in the time from onset of PsA symptoms to diagnosis. The median time from onset of symptoms to diagnosis was 1.2 years, and a significant proportion of patients (approximately one-third) experienced delays of $>4$ years. The delay in diagnosis could impact long-term clinical and patient-reported outcomes; delays as short as $\geq 6$ months have been shown to contribute to structural joint damage and poor physical function [7], and similar results have been observed for delays of $\geq 1$ and $\geq 2$ years $[8,9]$. Of note, the time from symptom onset to diagnosis was significantly longer for patients enrolled by dermatologists than for those enrolled by rheumatologists ( 2.6 vs. 1.0 years). This may be because rheumatologists are more familiar with the joint-related symptoms associated with PsA, which have some similarities with those observed in other forms of arthritis such as rheumatoid arthritis [20]. Patients who presented with skin and joint symptoms simultaneously were more quickly diagnosed than those first presenting with either symptom type alone, probably because multifaceted symptoms and signs aided diagnosis.

Another important finding of the study was the variation in disease activity and disease burden across enrolling specialties and geographic regions. Patients enrolled in the dermatology setting had higher scores on measures of skin involvement and enthesitis than did those from the rheumatology setting, possibly because patients displaying more severe skin symptoms were more likely to be referred to a dermatologist. In addition, patients in western areas of the USA displayed less severe disease than those in central or eastern areas. The reasons for this are unclear and warrant further investigation. However, possible reasons could include differences in formulary restrictions and insurance policies and the availability of rheumatologists and/or dermatologists across the different regions. These factors may also have influenced the time to receiving treatment with csDMARDs and bDMARDs, although these parameters did not show significant differences across regions.

A multidisciplinary approach has been recommended by both EULAR and GRAPPA to improve the diagnosis and management of PsA $[10,11]$. Co-management by rheumatologists and dermatologists has been shown to improve disease activity and quality-of-life outcomes in these patients $[21,22]$. Studies have shown that psoriasis usually precedes the development of PsA by several years and that the prevalence of undiagnosed PsA in patients with psoriasis is high in the dermatology setting [23-27]. Our findings that patients enrolled in the dermatology setting experience a longer delay to diagnosis than those enrolled in the 
Table 3 Current disease activity and disease burden by geographic region

\begin{tabular}{|c|c|c|c|c|}
\hline Measure & Eastern $(n=348)$ & Central $(n=94)$ & Western $(n=71)$ & $p$ value $^{\mathrm{a}}$ \\
\hline TJC68 & $8.8 \pm 10.7$ & $10.6 \pm 12.3$ & $5.7 \pm 7.4$ & 0.012 \\
\hline SJC66 & $4.0 \pm 5.7$ & $4.6 \pm 9.2$ & $1.2 \pm 2.1$ & 0.001 \\
\hline Enthesitis based on LEI & $1.3 \pm 1.7$ & $0.9 \pm 1.6$ & $0.4 \pm 1.1$ & $<0.001$ \\
\hline Dactylitis count & $0.7 \pm 1.6$ & $0.9 \pm 1.7$ & $0.3 \pm 0.7$ & 0.039 \\
\hline BASDAI & $4.9 \pm 3.2$ & $4.8 \pm 3.2^{\mathrm{b}}$ & $2.7 \pm 2.8$ & $<0.001$ \\
\hline BSA $(\%)$ & $6.3 \pm 12.6$ & $6.1 \pm 13.8$ & $3.6 \pm 5.3$ & 0.230 \\
\hline Psoriatic nail count & $1.9 \pm 3.2^{\mathrm{c}}$ & $1.5 \pm 2.6^{\mathrm{d}}$ & $2.6 \pm 3.1^{\mathrm{e}}$ & 0.103 \\
\hline PASI & $3.8 \pm 6.6^{\mathrm{f}}$ & $4.0 \pm 6.3^{\mathrm{g}}$ & $3.5 \pm 3.6^{\mathrm{h}}$ & 0.884 \\
\hline PtGA at the time of diagnosis & $6.8 \pm 2.8^{\mathrm{i}}$ & $6.0 \pm 2.9^{j}$ & $5.6 \pm 2.9$ & $<0.001$ \\
\hline PtGA during last visit & $4.8 \pm 2.9^{\mathrm{k}}$ & $5.4 \pm 3.1^{1}$ & $3.5 \pm 2.8^{\mathrm{m}}$ & 0.001 \\
\hline HAQ-DI & $0.9 \pm 0.7$ & $0.8 \pm 0.7$ & $0.4 \pm 0.5$ & $<0.001$ \\
\hline SF12v2 PCS & $39.9 \pm 9.8^{\mathrm{n}}$ & $40.4 \pm 11.3$ & $46.5 \pm 10.9$ & $<0.001$ \\
\hline SF12v2 MCS & $47.1 \pm 11.5^{\mathrm{n}}$ & $47.1 \pm 10.6$ & $48.0 \pm 11.7$ & 0.829 \\
\hline DLQI & $6.0 \pm 6.5^{\mathrm{n}}$ & $6.6 \pm 6.3^{\circ}$ & $6.1 \pm 6.0$ & 0.753 \\
\hline
\end{tabular}

Bold text indicates significant differences

Data are presented as mean \pm standard deviation unless otherwise indicated

BASDAI Bath Ankylosing Spondylitis Disease Activity Index, BSA body surface area with psoriasis, DLQI Dermatology Life Quality Index, $H A Q-D I$ Health Assessment Questionnaire-Disability Index, LEI Leeds Enthesitis Index, MCS Mental Component Score, PASI Psoriasis Area and Severity Index, PCS Physical Component Score, PtGA Patient Global Assessment of disease, SF12v2 Short Form 12-item health survey version 2.0, SJC66 swollen joint count in 66 joints, TJC68 tender joint count in 68 joints

${ }^{\mathrm{a}} p$ value from one-way analysis of variance

${ }^{\mathrm{b}} n=86$

${ }^{\mathrm{c}} n=312$

${ }^{\mathrm{d}} n=87$

${ }^{\mathrm{e}} n=69$

${ }^{\mathrm{f}} n=306$

$\mathrm{g}_{n=85}$

${ }^{\mathrm{h}} n=68$

${ }^{\mathrm{i}} n=345$

$\mathrm{j}_{n}=87$

$\mathrm{k}_{n}=298$

${ }^{1} n=57$

$\mathrm{m}_{n=51}$

${ }^{\mathrm{n}} n=347$

${ }^{\circ} n=90$

rheumatology setting support the use of a multidisciplinary approach for the management of PsA.

The main limitation of this study was its observational nature. Also, the full analysis set was based on confirmation of PsA by the rheumatologist only, so the data collected may not be reflective of the real-world management of patients across the USA. Similarly, categorization and analysis of data by enrolling specialty may not have adequately captured the complexity of the patient journey, such as how long the patient had been seeing that specialist, when and whether patients had prior care, and who made the initial diagnosis in patients with established PsA. In addition, conclusions are limited for some of the investigated parameters, as missing data restricted sample sizes and prevented statistical comparisons.

In summary, our results show that clinical specialty setting and geographic region can affect the timing and choice of disease management steps as well as disease activity and disease burden in patients with PsA in the USA. There was a substantial delay in the time from symptom onset to diagnosis, and this was significantly longer for patients enrolled by dermatologists than for those enrolled by rheumatologists. Clinical specialty setting also affected the severity of skin symptoms, and patients from eastern and central USA tended to have more severe disease than those from the western region. The delay in disease diagnosis and high disease 
activity in these patients highlights the need for a multidisciplinary approach in the diagnosis and management of PsA.

Acknowledgements AbbVie and the authors thank all study investigators for their contributions and the patients who participated in this study. Medical writing support was provided by John Ewbank, Ph.D., of 2 the Nth, funded by AbbVie.

\section{Compliance with Ethical Standards}

Funding AbbVie funded the LOOP study, contributed to its design, and participated in data collection, analysis, and interpretation of the data, and in writing, review, and approval of the publication.

Data sharing AbbVie is committed to responsible data sharing regarding the clinical trials we sponsor. Access is provided to anonymized patient and trial-level data (analysis datasets) as well as other information (e.g., protocols and clinical study reports) from AbbVie-sponsored phase II-IV global interventional clinical trials conducted in patients (completed as of May 2004, for products and indications approved in either the USA or the EU) as long as the trials are not part of an ongoing or planned regulatory submission. This includes requests for clinical trial data for unlicensed products and indications. Access to these clinical trial data can be requested by any qualified researchers who engage in rigorous, independent scientific research and will be provided following review and approval of a research proposal and statistical analysis plan and execution of a data-sharing agreement. Data requests can be submitted at any time, and the data will be accessible for 12 months, with possible extensions considered. For more information on the process or to submit a request, visit the following link: https://www.abbvie.com/our-science/clinical-trials/clinical-trial s-data-and-information-sharing/data-and-information-sharing-withqualified-researchers.html.

Conflict of interest P.J. Mease has received research grants, consulting fees, and/or speaker's fees from AbbVie, Amgen, Bristol-Myers Squib, Celgene, Galapagos, Genentech, Gilead, Janssen, Lilly, Novartis, Pfizer, Sun Pharma, and UCB. B. Lockshin has received research grants, consulting fees, and speaker's fees from AbbVie, Janssen, Lilly, and Novartis; has received research grants from Celgene and Strata; and is on the board of directors for the National Psoriasis Foundation. C. Liu has served as a speaker, on an advisory board, and/or as an investigator for AbbVie, Celgene, Janssen, Lilly, Novartis, Ortho, Regeneron, and Sanofi. E. Siegel has received research grants and acted as a speaker for and/or received consulting fees from AbbVie, Amgen, Celgene, Janssen, Lilly, Sanofi, and UCB. H. Richmond has received a research grant from AbbVie. M. Wu, L. Chen, and K. Douglas are employees of AbbVie and may own stock/options.

Ethical approval All procedures performed in studies involving human participants were in accordance with the ethical standards of the institutional and/or national research committees and with the Declaration of Helsinki. All patients provided written informed consent.

Open Access This article is distributed under the terms of the Creative Commons Attribution-NonCommercial 4.0 International License (http://creativecommons.org/licenses/by-nc/4.0/), which permits any noncommercial use, distribution, and reproduction in any medium, provided you give appropriate credit to the original author(s) and the source, provide a link to the Creative Commons license, and indicate if changes were made.

\section{References}

1. Van den Bosch F, Coates L. Clinical management of psoriatic arthritis. Lancet. 2018;391(10136):2285-94.

2. Ritchlin CT, Colbert RA, Gladman DD. Psoriatic arthritis. N Engl J Med. 2017;376(10):957-70.

3. Gladman D, et al. Psoriatic arthritis: epidemiology, clinical features, course, and outcome. Ann Rheum Dis. 2005;64(Suppl. 2):ii14-7.

4. Dhir V, Aggarwal A. Psoriatic arthritis: a critical review. Clin Rev Allergy Immunol. 2013;44(2):141-8.

5. Moll J, Wright V. Psoriatic arthritis. Semin Arthritis Rheum. 1973;3(1):55-78.

6. Haddad A, Zisman D. Comorbidities in patients with psoriatic arthritis. Rambam Maimonides Med J. 2017;8(1):e0004.

7. Haroon M, Gallagher P, FitzGerald O. Diagnostic delay of more than 6 months contributes to poor radiographic and functional outcome in psoriatic arthritis. Ann Rheum Dis. 2015;74(6):1045-50.

8. Tillett W, et al. Smoking and delay to diagnosis are associated with poorer functional outcome in psoriatic arthritis. Ann Rheum Dis. 2013;72(8):1358-61.

9. Gladman DD, et al. Do patients with psoriatic arthritis who present early fare better than those presenting later in the disease? Ann Rheum Dis. 2011;70(12):2152-4.

10. Coates LC, et al. Group for Research and Assessment of Psoriasis and Psoriatic Arthritis 2015 treatment recommendations for psoriatic arthritis. Arthritis Rheum. 2016;68(5):1060-71.

11. Gossec L, et al. European League Against Rheumatism (EULAR) recommendations for the management of psoriatic arthritis with pharmacological therapies: 2015 update. Ann Rheum Dis. 2016;75(3):499-510.

12. Singh JA, et al. Special Article: 2018 American College of Rheumatology/National Psoriasis Foundation guideline for the treatment of psoriatic arthritis. Arthritis Care Res (Hoboken). 2019;71(1):2-29.

13. Acosta Felquer ML, et al. Drug therapies for peripheral joint disease in psoriatic arthritis: a systematic review. J Rheumatol. 2014;41(11):2277-85.

14. Eder L, et al. Tumour necrosis factor alpha blockers are more effective than methotrexate in the inhibition of radiographic joint damage progression among patients with psoriatic arthritis. Ann Rheum Dis. 2014;73(6):1007-11.

15. Jones G, Crotty M, Brooks P. Interventions for psoriatic arthritis. Cochrane Database Syst Rev. 2000;3:Cd000212.

16. Kaltwasser JP, et al. Efficacy and safety of leflunomide in the treatment of psoriatic arthritis and psoriasis: a multinational, double-blind, randomized, placebo-controlled clinical trial. Arthritis Rheum. 2004;50(6):1939-50.

17. Kingsley GH, et al. A randomized placebo-controlled trial of methotrexate in psoriatic arthritis. Rheumatology. 2012;51(8):1368-77.

18. Goulabchand R, et al. Effect of tumour necrosis factor blockers on radiographic progression of psoriatic arthritis: a systematic review and meta-analysis of randomised controlled trials. Ann Rheum Dis. 2014;73(2):414-9.

19. Ornbjerg LM, et al. Impact of tumour necrosis factor inhibitor treatment on radiographic progression in rheumatoid arthritis patients in clinical practice: results from the nationwide Danish DANBIO registry. Ann Rheum Dis. 2013;72(1):57-63.

20. McArdle A, Pennington S, FitzGerald O. Clinical features of psoriatic arthritis: a comprehensive review of unmet clinical needs. Clin Rev Allergy Immunol. 2018;55(3):271-94.

21. Luchetti MM, et al. Clinical outcomes and feasibility of the multidisciplinary management of patients with psoriatic arthritis: 
2-year clinical experience of a dermo-rheumatologic clinic. Clin Rheumatol. 2018;37(10):2741-9.

22. Luelmo J, et al. A report of 4 years of experience of a multidisciplinary unit of psoriasis and psoriatic arthritis. Reumatol Clin. 2014;10(3):141-6.

23. Villani A, et al. Prevalence of undiagnosed psoriatic arthritis among psoriasis patients: systematic review and meta-analysis. J Am Acad Dermatol. 2015;73(2):242-8.

24. Choi JW, et al. Could psoriatic arthritis be easily diagnosed from current suspicious physical findings in the dermatology clinic? Ann Dermatol. 2017;29(1):48-54.
25. Mease PJ, et al. Prevalence of rheumatologist-diagnosed psoriatic arthritis in patients with psoriasis in European/North American dermatology clinics. J Am Acad Dermatol. 2013;69(5):729-35.

26. Papadavid E, et al. Prevalence of psoriatic arthritis and its correlates among patients with psoriasis in Greece: results from a large retrospective study. J Eur Acad Dermatol Venereol. 2016;30(10):1749-52.

27. Spelman L, et al. Frequency of undiagnosed psoriatic arthritis among psoriasis patients in Australian dermatology practice. $\mathrm{J}$ Eur Acad Dermatol Venereol. 2015;29(11):2184-91. 